\title{
Do natural antibodies have a detrimental effect after kidney transplantation?
}

\author{
Arnaud Del Bello ${ }^{1}$, Nassim Kamar ${ }^{1,2,3}$ \\ ${ }^{1}$ Department of Nephrology and Organ Transplantation, CHU de Toulouse - Rangueil, Toulouse, France; ${ }^{2}$ Paul Sabatier University, Toulouse, \\ France; ${ }^{3}$ INSERM U1043, IFR-BMT, Purpan CHU, Toulouse, France \\ Correspondence to: Arnaud Del Bello, MD; Nassim Kamar, MD, PhD. Department of Nephrology and Organ Transplantation, CHU de Toulouse - \\ Rangueil, TSA 50032, 31059 Toulouse Cedex 9, France. Email: delbello.a@chu-toulouse.fr; kamar.n@chu-toulouse.fr. \\ Comment on: See SB, Aubert O, Loupy A, et al. Post-Transplant Natural Antibodies Associate with Kidney Allograft Injury and Reduced Long-Term \\ Survival. J Am Soc Nephrol 2018;29:1761-70.
}

Submitted Jul 05, 2018. Accepted for publication Jul 10, 2018.

doi: 10.21037/atm.2018.07.10

View this article at: http://dx.doi.org/10.21037/atm.2018.07.10

Acute and chronic antibody mediated rejection (AMR) are currently the main causes of allograft loss after kidney transplantation $(1,2)$. Anti-human leukocyte antigen (HLA) donor-specific antibodies (DSAs) are the principal antibodies that cause antibody-mediated rejections. These DSAs have been characterized and it has been shown that some subclasses of anti-HLA IgG antibodies, i.e., the IgG1 and IgG3 subclasses, are more harmful than other subclasses $(3,4)$. Similarly, complement-binding DSAs have been associated with a lower kidney-allograft survival rate compared to non-complement-binding DSAs $(5,6)$. Over the past few years, studies have been conducted on the impact of non-HLA antibodies on kidney allograft survival. Antiangiotensin II type 1 receptor (7), anti-endothelial cell (8), and antiperlecan antibodies (LG3) (9) were shown to be associated with acute and chronic AMR, with a potential synergistic effect with DSAs (10).

Natural antibodies (Nabs) were described more than 50 years ago (11). Their characteristics are low affinity, low valency but high avidity and poly-recognition (12). However, their pathogenicity remains unclear. IgM and IgG Nabs have been detected in the blood of healthy donors at low titers (13). Conversely, IgG Nabs have been detected at high titers in auto-immune diseases such as systemic lupus erythematosus (14). In transplant patients, Nabs, which are polyreactive antibodies that can react with several antigens such as DNA, apoptotic cells or oxidation-related epitopes, are suspected of inducing AMR and of having a negative impact on graft survival. A group from New York noted the development, during AMR episodes, of polyreactive antibodies that are cross-reactive to apoptotic cells. IgG reactivity to apoptotic cells was significantly higher in the sera of 20 kidney-transplant patients with AMR compared to 20 other kidney-transplant patients with stable kidney function. In addition, total IgG purified from AMR patients increased complement-activating properties compared to IgG from non-AMR patients (15). In a retrospective study, the same group assessed the presence of polyreactive antibodies that are cross-reactive to apoptotic cells in pretransplant sera from 300 kidney-transplant patients. They found that high pre-transplant IgG reactivity to apoptotic cells was associated with an increased risk of late graft loss. This was also observed after excluding patients with high reactivity to HLA molecules (16). Later, they reported that elevated pre-transplant IgG Nabs that react to apoptotic cells and to malondialdehyde (MDA), a generic oxidized epitope, were associated with the development of primary heart allograft dysfunction, especially in patients with a ventricular assist device (17).

Quite recently, a collaborative study between the New York group and the Necker Hospital (Paris, France) group wished to assess the effect of Nabs in a large cohort of kidney-transplant patients (18). They studied the reactivity of IgG Nabs to MDA, a product of lipid peroxidation resulting from oxidative stress that binds to proteins and lipids creating neoepitopes recognized by Nabs. Nabs were assessed before and during the first year after transplantation in a very well characterized cohort of $635 \mathrm{ABO}$-compatible, 
negative complement-dependent cytotoxicity crossmatch kidney-transplant patients from Necker Hospital. The generation of Nabs was defined by an increase in serum reactivity to MDA of at least $50 \%$ between the pretransplant and the post-transplant serum (18). The analysis was blinded for clinical and histological outcomes. Sixtysix of the 635 patients $(10.4 \%)$ were considered to have de novo Nabs. After a median follow-up of 7.6 \pm 2.8 years, patients with Nabs during the first year, with or without detectable DSAs, had significantly worse graft survival compared to those without Nabs or DSAs. Histological findings for protocol kidney biopsies performed 1 year after transplantation, showed a significantly higher rate of microvascular inflammation, transplant glomerulopathy, and C4d deposition in patients with Nabs, with or without DSAs, compared to those with neither Nabs nor DSAs. It is interesting to note that the presence of DSA with an MFI $\geq 6,000$ and the development of Nabs were independent predictive factors for graft loss. These data clearly suggest that the detection of Nabs is associated with impaired kidney histology and decreased kidney allograft survival.

The study described above was quite thoroughly conducted by two expert groups in the field of AMR. However, there are still some unanswered questions. The mechanism of action of Nabs is currently unknown. The data by See et al. which showed that kidney allograft survival is significantly worse in patients with Nabs, with or without DSAs, and the fact that a significantly higher proportion of patients with only Nabs have increased C4d deposition, indicate that there is a direct and detrimental effect of Nabs on kidney allografts and that this effect is complement-dependent. In addition, the fact that patients with both DSAs and Nabs have the worst outcome suggests a synergistic effect of the two antibodies. Patients' sera were verified to ensure that the other antibodies that can induce AMR, such as anti-angiotensin II type 1 receptor, anti-endothelial cell, or anti-MICA antibodies were not detectable and did not contribute to histological findings and poor kidney-allograft outcomes. It would also be interesting to determining the subclasses of Nabs and to assess whether or not they bind to the complement. In fact, some IgM Nabs were found to abrogate inflammation mediated by microorganisms (19), which highlights the complexity of regulating mechanisms and the need for a better comprehension of the role of Nabs.

It was previously suspected that Nabs were involved in multiple biological processes including infection, B cell homeostasis, inflammation, atherosclerosis and autoimmunity (20). Hence, it was considered that several situations such as ischemia reperfusion injuries, episodes of T-cell mediated acute rejection and infections could induce oxidative stress, ultimately leading to the generation of Nabs that react with MDA. For instance, in the study by See et al., in comparison to patients without Nabs, more of those with Nabs had received kidneys from extendedcriteria deceased donors, had prior transplantation, and were grafted with DSAs. This could be responsible for increased oxidative stress that could generate Nabs. In this study, the timing between transplantation and the generation of Nabs, a history of acute rejection, a history of infections, and the interval between acute rejection episodes (if any) or infectious complications (if any) are unknown. These data could help to improve the understand of what causes Nabs to appear. In addition, it is uncertain whether or not the immunosuppressive regimen which mainly consists of T-cell depleting agents, rituximab, and apheresis affects the development of Nabs.

In summary, See et al. elegantly showed that the presence of Nabs which react to MDA is associated with decreased kidney allograft survival, microvascular histological lesions, histological features of transplant glomerulopathy, and C4d deposition. However, it is still unknown whether Nabs are responsible for these findings or whether the generation of Nabs is due to kidney aggression not caused by DSAs and which has a detrimental effect on the grafted kidney. Further studies are required to improve the understanding of the cause of Nabs and their mechanism of action, as well as to confirm the reported data.

\section{Acknowledgements}

None.

\section{Footnote}

Conflicts of Interest: The authors have no conflicts of interest to declare.

\section{References}

1. Colvin RB, Smith RN. Antibody-mediated organ allograft rejection. Nat Rev Immunol 2005;5:807-17.

2. Orandi BJ, Luo X, Massie AB, et al. Survival Benefit with kidney transplant from HLA incompatible live-donors. $\mathrm{N}$ Engl J Med 2016;374:940-50.

3. Cicciarelli JC, Lemp NA, Chang Y, et al. Renal Transplant 
Patients Biopsied for Cause and Tested for C4d, DSA, and IgG Subclasses and C1q: Which Humoral Markers Improve Diagnosis and Outcomes? J Immunol Res 2017;2017:1652931.

4. Hamdani G, Goebel JW, Brailey P, et al. IGG3 antiHLA donor-specific antibodies and graft function in pediatric kidney transplant recipients. Pediatr Transplant 2018:e13219.

5. Loupy A, Lefaucheur C, Vernerey D, et al. Complementbinding anti-HLA antibodies and kidney-allograft survival. New Engl J Med 2013;369:1215-26.

6. Sicard A, Ducreux S, Rabeyrin M, et al. Detection of C3dbinding donor-specific anti-HLA antibodies at diagnosis of humoral rejection predicts renal graft loss. J am Soc Nephrol 2015;26:457-67.

7. Dragun D, Müller DN, Bräsen JH, et al. Angiotensin II type 1-receptor activating antibodies in renal allograft rejection. N Engl J Med 2005;352:558-69.

8. Philogene MC, Zhou S, Lonze BE, et al. Pre-transplant Screening for Non-HLA Antibodies: Who should be Tested? Hum Immunol 2018;79:195-202.

9. Cardinal H, Dieudé M, Brassard N, et al. Antiperlecan antibodies are novel accelerators of immune-mediated vascular injury. Am J Transplant 2013;13:861-74.

10. Taniguchi M, Rebellato LM, Cai J, et al. Higher risk of kidney graft failure in the presence of anti-angiotensin II type 1 receptor antibodies. Am J Transplant 2013;13:2577-89.

11. Boyden SV. Natural Antibodies and the immune response. Adv Immunol 1966;5:1-28.

Cite this article as: Del Bello A, Kamar N. Do natural antibodies have a detrimental effect after kidney transplantation? Ann Transl Med 2018;6(16):316. doi: 10.21037/atm.2018.07.10
12. Panda S, Ding JL. Natural Antibodies bridge innate and adaptive immunity. J Immunol 2015;194:13-20.

13. Zorn E. New Insights on innate B-cell immunity in transplantation. Xenotransplantation 2018;25:e12417.

14. Cabral AR, Alarcon-Segovia D. Autoantibodies in systemic lupus erythematosus. Curr Opin Rheumatol 1998;10:409-16.

15. Porcheray F, Fraser JW, Gao B, et al. Polyreactive antibodies developing amidst humoral rejection of human kidney grafts bind apoptotic cells and activate complement. Am J Transplant 2013;13:2590-600.

16. Gao B, Moore C, Porcheray F, et al. Pretransplant IgG reactivity to apoptotic cells correlates with late kidney allograft loss. Am J Transplant 2014;14:1581-91.

17. See SB, Clerkin KJ, Kennel PJ, et al. Ventricular assist device elicits serum natural IgG that correlates with the development of primary graft dysfunction following heart transplantation. J Heart Lung Transplant 2017;36:862-70.

18. See SB, Aubert O, Loupy A, et al. Post-transplant natural antibodies associate with kidney allograft injury and reduced long-term survival. J Am Soc Nephrol 2018;29:1761-70.

19. Lobo PI. Role of Natural IgM autoantibodies (IgMNAA) and IgM anti-leucocytes antibodies (IgM-ALA) in regulating inflammation. Curr Top Microbiol Immunol 2017;408:89-117.

20. Ehrenstein MR, Notley CA. The importance of natural IgM: scavenger, protector, and regulator. Nat Rev Immunol 2010;10:778-86. 